\title{
Reflets
}

Revue d'intervention sociale et communautaire

OTERO, Marcelo, et Shirley ROY (dirs.) (2013). Qu'est-ce qu'un problème social aujourd'hui ? Repenser la non-conformité. Collection Problèmes sociaux et interventions sociales, Presses de l’Université du Québec, 394 p.

\section{Jonathan Binet}

Volume 22, numéro 1, printemps 2016

URI : https://id.erudit.org/iderudit/1037172ar

DOI : https://doi.org/10.7202/1037172ar

Aller au sommaire du numéro

Éditeur(s)

Reflets, Revue d'intervention sociale et communautaire

ISSN

1203-4576 (imprimé)

1712-8498 (numérique)

Découvrir la revue

Citer ce compte rendu

Binet, J. (2016). Compte rendu de [OTERO, Marcelo, et Shirley ROY (dirs.) (2013).

Qu'est-ce qu'un problème social aujourd'hui ? Repenser la non-conformité.

Collection Problèmes sociaux et interventions sociales, Presses de l'Université

du Québec, 394 p.] Reflets, 22(1), 230-237. https://doi.org/10.7202/1037172ar 


\section{Qu'est-ce qu'un problème social aujourd'hui? Repenser la non- conformité}

OTERO, Marcelo, et Shirley ROY (dirs.) (2013). Collection Problèmes sociaux et interventions sociales, Presses de l'Université du Québec, 394 p.

par Jonathan Binet, M.S.S.

Professeur à temps partiel et doctorant en service social

École de service social, Université d'Ottawa

La désignation des problèmes sociaux, comme la désignation des populations qui les incarnent et la formulation des modalités d'intervention qui visent à y répondre, varie selon les contextes et les époques. Ainsi, toute société dessine, en fonction de la normativité sociale qui la traverse, ce qui pour elle pose problème et les manières d'y remédier. Là est toute la pertinence de cet ouvrage collectif dans lequel des auteures et auteurs revisitent les manières dont on a historiquement pensé les problèmes sociaux, pour mettre à jour leur utilité et, ainsi, comprendre et expliquer ceux d'aujourd'hui. En effet, on ne peut pas penser les problèmes sociaux contemporains sans considérer les transformations normatives qui se manifestent dans nos sociétés depuis les trente dernières années. Ces transformations de la normativité sociale, dont les causes sont fortement discutées en sciences sociales, ont contribué à redéfinir les partages entre le normal et l'anormal, le conforme et le non-conforme, le tolérable et l'intolérable. Ainsi, ces transformations normatives ont rendu obsolète la désignation de certains phénomènes sociaux, tels que l'enfance illégitime et le divorce, à titre de problèmes sociaux et 
ont permis, au contraire, de désigner comme posant problème certains autres phénomènes, tels que la violence conjugale et l'hyperactivité. Dans ce même ordre d'idées, les modalités d'intervention qui ciblent ce ou ceux qui posent problème se sont transformées et multipliées. Dans les sociétés contemporaines, il est possible de repérer une pluralité de modalités d'intervention parfois compréhensives, réformatrices, contrôlantes ou punitives qui visent à agir sur ce que l'on désigne comme des problèmes sociaux et sur les populations qui les incarnent. Sans être forcément nouvelles, ces modalités d'intervention se superposent, se hiérarchisent et s'organisent autour de problématiques bien circonscrites. Or, plutôt que d'adopter ces explications et ces regards « catégoriel[s], substantialiste [s], psychologisant $[\mathrm{s}]$ et parfois franchement folklorisant $[\mathrm{s}]$ » (quatrième de couverture), les auteures et auteurs de cet ouvrage proposent en quatre parties des analyses transversales cherchant à lier les récentes transformations sociétales à ces explications et regards.

La première partie, intitulée Dynamiques et rhétoriques, est divisée en quatre chapitres qui cherchent à déchiffrer les manières dont sont rationalisés les problèmes sociaux et les modalités d'intervention qui les ciblent. Dans le premier chapitre, Luc Van Campenhoudt remet en question l'idée d'une "idéologie consensualiste»(p. 33) qui s'impose aux actrices et acteurs qui agissent sur les problèmes sociaux et les populations qui les incarnent. L'auteur met à l'écart l'idée d'un complot en soutenant celle d'un «malentendu qui fonctionne » (p. 21). Selon l'auteur, les malentendus sont inhérents aux rapports sociaux dans lesquels sont définis les problèmes sociaux et les modalités d'intervention qui les ciblent. Dans la sphère des politiques publiques, cela implique que la construction des problèmes sociaux, par les dirigeantes et dirigeants, donne lieu à des distorsions sur les différents paliers d'intervention, ce qui n'est pas mauvais en soi. Dans le deuxième chapitre, Isabelle Astier met à jour la nouvelle grammaire de l'action publique, centrée sur l'accompagnement, l'activation et la responsabilisation. L'auteure soutient que les politiques publiques, sous l'ordre du nouveau management public, font de plus en plus la promotion d'une figure de l'individu responsable 
et participant. Ces nouvelles orientations normatives de l'action publique transforment l'intervention sociale au nom d'un principe de proximité et encouragent la multiplication de nouvelles figures professionnelles (travailleuses et travailleurs de rue, médiatrices et médiateurs, etc.). Ces professionnelles et professionnels du proche accompagnent l'individu dans un travail sur soi et l'incitent à agir plus qu'ils ne le réparent ou ne le libèrent. Ainsi prendrait place une réinstitutionnalisation dans laquelle de nouvelles normes (liberté, responsabilité, autonomie, projet, travail sur soi, etc.) baliseraient le travail des institutions et celui de leurs actrices et acteurs. Le travail social s'en verrait transformé dans l'optique où il ne servirait plus autant d'intermédiaire entre des valeurs générales et des individus que d'accompagnateur de ces derniers dans la construction de leur trajectoire biographique. Dahlia Namian aborde dans le troisième chapitre "La politique de la présentation des problèmes sociaux " (p. 57). À partir de sa thèse doctorale sur l'itinérance et la fin de vie, elle illustre comment la dynamique de décalage et de convulsion discutée dans les sciences sociales s'est introduite dans sa démarche sous la forme de deux procédés : l'ironie et le transfert. Son intention est alors de problématiser le rapport entre la recherche sur les problèmes sociaux et ses enjeux politiques en y insérant volontairement et explicitement des décalages et en laissant émerger des discours critiques. Le procédé de l'ironie permet à la chercheuse ou au chercheur d'introduire sur les plans de la rhétorique (style d'écriture) et de la méthode (comparaison, analogie, etc.) un décalage entre la réalité sociale et la manière dont elle est présentée. Notamment, l'auteure montre comment ce procédé lui a permis d'entrevoir la "vie moindre " (p. 63), mais aussi de redéfinir les manières dont on parle de l'itinérance et de la vie, et de critiquer l'humanisme. Le procédé du transfert permet quant à lui de déplacer une image ou une idée de son champ d'application initial à un autre. Ainsi, le transfert permet de redonner sens à des objets à l'aide d'une stratégie langagière, d'un jeu de mots. En empruntant notamment le terme "couveuse " (p. 66) aux domaines de la médecine et de l'entrepreneuriat, l'auteure en est venue, par exemple, à désigner par le même mot les dispositifs qui permettent aux individus incarnant les problèmes 
sociaux de compléter leur processus d'individuation. Dans le quatrième chapitre,Vivianne Châtel conclut cette première partie du livre en discutant des frontières et des enjeux qu'elles posent à la compréhension des problèmes sociaux. Les frontières forment des espaces, imaginaires et physiques, dans lesquels sont partagés en catégories les individus (eux/nous, dignes/indignes, normaux/ anormaux, etc.) et dessinent ainsi la carte mobile des problèmes sociaux. L'auteure soutient que la question des frontières pose des enjeux de taille en constituant des catégories qui permettent de définir de manière englobante les individus et d'invisibiliser leurs caractéristiques humaines.

La deuxième partie, intitulée Ambivalences et interrogations, est composée de cinq chapitres. Les auteures et auteur cherchent à illustrer les ambiguités qui traversent les disciplines, les contextes et l'histoire, et qui interpellent les manières dont sont séparés le conforme du non-conforme comme les modalités d'intervention qui visent à y répondre. Ainsi, dans le cinquième chapitre, MarieChantal Doucet montre que les principes du rétablissement qui sont à la base des services de santé mentale comportent des limites et des enjeux. Elle soutient notamment que ces principes, même s'ils se présentent sous une allure non directive, témoignent d'un retour au traitement moral des malades en exigeant d'eux qu'ils se positionnent comme des individus autonomes et responsables. Ce faisant, les postulats du rétablissement écarteraient les histoires individuelles et la réflexivité des actrices et acteurs. À cet effet, l'auteure propose que les réflexions à propos des problèmes sociaux doivent intégrer les histoires individuelles ainsi que reconnaitre la capacité des individus à donner un sens à leurs expériences et aux épreuves rencontrées. Dans le sixième chapitre, Nathalie Mondain s'intéresse aux approches démographiques ainsi qu'aux conséquences de ces modes de problématisation des populations lorsqu'elles sont appliquées aux populations des pays dits en développement. Elle souligne la nécessité de revisiter les enjeux éthiques soulevés par le transfert des catégories problématiques et des modalités d'intervention des sociétés occidentales dans les pays dits en développement pour éviter qu'elles participent davantage à reproduire les problèmes qu'elles ciblent. En ce sens, l'auteure 
se demande si, avec les approches démographiques, la population constitue « un problème social ou un enjeu de pouvoir permettant la reproduction de tout un système - le développement international - qui fonctionne sur une appréhension erronée des enjeux autour desquels il s'est constitué depuis maintenant plus d'un demi-siècle "(p.121). Dans le septième chapitre, Dominic Dubois aborde la question du phénomène "trans " et montre en analysant les registres médicaux et sociaux qu'il s'agit d'un intéressant révélateur des transformations qui traversent les sociétés contemporaines. Dans le huitième chapitre, Carolyne Grimard propose de dépasser la figure stéréotypée du clochard pour entrevoir la complexité et la diversité de l'itinérance. En s'intéressant aux interactions entre les refuges et les hommes qui incarnent l'itinérance, l'auteure propose une typologie permettant de dessiner ce à quoi le terme réfêre bien souvent. Loin d'être un phénomène homogène, l'itinérance regroupe aujourd'hui une pluralité de figures qui entrent en interaction avec les services des refuges et leur rationalité institutionnelle. Certains hommes adoptent le refuge à titre de milieu de vie et n'envisagent pas de le quitter. D'autres effectuent des va-et-vient entre le refuge et un autre milieu de vie. Pour plusieurs raisons (manque de ressources, obstacles, etc.), leurs expériences sont marquées paradoxalement par une mobilisation de soi vers la sortie du refuge, mais aussi par leur attachement à ce dernier duquel ils ne peuvent se priver. Finalement, d'autres hommes se mobilisent, s'appuient sur les ressources qui se présentent à eux et posent des actions qui leur permettent de quitter la vie du refuge. À cet égard, l'auteure soutient que les services des refuges sont souvent mieux adaptés à ces individus passagers qu'aux autres. Les coûts associés à la prise en charge des individus les plus fragiles et vulnérables sont trop élevés, et les refuges ne réussissent tout simplement pas à formuler des réponses adéquates à leurs situations. Les refuges gèrent néanmoins "un problème social insoluble " (p. 165) en permettant à certains de quitter l'univers de la rue et en soulageant les autres et la société. Dans le neuvième chapitre, Valérie de Courville Nicol conclut cette deuxième partie du livre en empruntant à la sociologie de la subjectivité des outils qui permettent de distinguer l'expérience 
subjective du danger de son expérience morale. Statuant que toute expérience d'un problème est une expérience du danger, elle met de l'avant l'idée que "l'expérience du danger comme problème moral est une condition de l'expérience du danger comme problème social " (p. 168). En ce sens, elle suggère de revisiter les liens entre problèmes sociaux et moralité qui sont fréquemment réfléchis à partir de l'impact des normes morales sur la problématisation.

La troisième partie, intitulée Émergences et constructions, comprend quatre chapitres qui offrent des réflexions sur les dynamiques sociales, institutionnelles et historiques dans lesquelles sont construits les problèmes sociaux. Dans le dixième chapitre, Johanne Collin s'intéresse aux questions de la perméabilité et du déplacement des frontières entre le normal et l'anormal. Elle cherche à expliquer le passage de la médicalisation à la pharmaceuticalisation. Dans sa réflexion, la médicalisation se présente comme un fruit issu de la convergence des valeurs importantes pour tous les groupes de la société que sont la fixation sur le risque et le désir d'une santé parfaite. Or, selon l'auteure, la médicalisation devrait être le départ d'une analyse plutôt que sa conclusion. Par ailleurs, la pharmaceuticalisation n'est pas que la continuation du déploiement de la médicalisation dans le social. Elle désigne le processus par lequel un médicament en vient à s'inscrire dans le quotidien des individus, à altérer leur identité ainsi que les dynamiques sociales. Dans le onzième chapitre, Céline Bellot, Marie-Ève Sylvestre et Bernard St-Jacques proposent une réflexion sur la possibilité d'adopter une posture de recherche marquée par l'engagement et porteuse de changement social. Pour se positionner ainsi, la chercheuse ou le chercheur doit, selon Bellot, Sylvestre et St-Jacques, faire preuve de façon continue d'une réflexivité éthique. En s'inspirant de leurs travaux, les auteures et auteur montrent, par exemple, comment la chercheuse ou le chercheur peut contribuer à mettre à jour la judiciarisation en tant que problème social. Dans le douzième chapitre, Martin Petitclerc rappelle que la construction des problèmes sociaux relève autant, sinon plus, des négociations entre les individus que de la réalité sociale elle-même. Les enjeux sociopolitiques marquent en ce sens 
la construction de ce qui pose problème. Dans le cas du système d'assurance-maladie du Québec, par exemple, les débats qui l'ont entouré illustrent comment la définition d'un problème social (la maladie) et des risques sur lesquels les individus doivent intervenir ne peut pas être remise en question sans considérer les valeurs de la " communauté politique» (p. 246). Nicolas Carrier conclut cette troisième partie du livre en soutenant, dans le treizième chapitre, que les chercheuses et chercheurs des sciences sociales doivent choisir entre des analyses objectiviste et constructiviste pour observer ce qui pose problème. En prenant l'exemple empirique des modes de problématisation de l'usage des drogues illicites et de leurs usagères et usagers, l'auteur souligne la difficulté à accepter la distinction entre les diagnostics du monde social et la réalité elle-même qui traverse l'exercice de problématisation.

La quatrième et dernière partie du livre, intitulée Relectures et nouvelles perspectives, dresse un bilan en trois chapitres des modes de problématisation de ce qui est non conforme et met à jour les voies pour ramener à l'avant-plan les réflexions sur les politiques publiques. Dans le quatorzième chapitre, Henri Dorvil revient sur les apports de l'École de Chicago à la compréhension contemporaine des problèmes sociaux en sociologie et en travail social.L'auteur essaie de dégager de cette relecture des perspectives d'avenir autour de trois thèmes : les réseaux sociaux, la privatisation et la nouvelle normativité. Dans le quinzième chapitre, Shirley Roy et Roch Hurtubise soutiennent que la difficulté à effectuer des analyses transversales explique l'immobilisme dans les réflexions sur les problèmes sociaux québécois. En s'inspirant de leurs travaux sur l'itinérance, Roy et Hurtubise montrent comment il est possible de faire preuve "d'efficacité théorique " (p. 346) et de mettre en jeu les différents points de vue (savoirs, expertises, disciplines, méthodologies, etc.) pour ainsi contribuer au renouvellement des connaissances sur un thème donné. Finalement, dans le seizième et dernier chapitre, Marcelo Otero argumente que l'analyse des problèmes sociaux nécessite un déplacement à l'égard de ce qui pose problème. Ce passage est celui de l'analyse des populations qui posent problème à une interrogation sur ce qui pose socialement problème. 
Tout compte fait, l'ouvrage Qu'est-ce qu'un problème social aujourd'hui? Repenser la non-conformité remet à l'avant-plan des réflexions sur les enjeux qui entourent la désignation des problèmes sociaux et des individus qui les incarnent et la formulation des modalités d'intervention. Dans cet ordre d'idées, l'ouvrage propose des pistes et des outils pour analyser autrement les manières dont sont désignés ce ou ceux qui posent problème et les méthodes d'intervention qui les ciblent.

Pour ceux qui s'intéressent à la question des populations vivant en contexte linguistique minoritaire, l'ouvrage incite notamment à se défaire des explications individualisantes et catégorielles et à réinterpréter les problèmes sociaux qui les concernent en lien avec les transformations de la normativité sociale. Les auteures et auteurs de cet ouvrage offrent, sous forme de boîte à outils, des instruments pertinents pour remettre en question les discours et les pratiques qui ciblent les populations vivant en contexte linguistique minoritaire et pour renouer avec les fondements critiques de la discipline du service social. 